J. Asiat. Soc. Bangladesh, Sci. 39(2): 173-182, December 2013

\title{
LIMNOLOGICAL STATUS OF TRIMOHINI BEEL OF RAJSHAHI, BANGLADESH
}

\author{
MD. AZIZUL ISLAM AND ABDULLAH HARUN CHOWDHURY ${ }^{1}$ \\ Limnology Laboratory, Department of Botany, Rajshahi University, Rajshahi \\ ${ }^{1}$ Environmental Science Discipline, Khulna University, Khulna
}

\begin{abstract}
A total of 38 zooplankton genera and 26 physico-chemical variables were recorded in Trimohini Beel. This beel marked as a medium level of polluted wetland based on the values of the redox characteristics i.e. $\mathrm{pH}, \mathrm{DO}, \mathrm{BOD}, \mathrm{COD}$, Eh and $\mathrm{rH}_{2}$, chlorides, nitrites, ammonium, phosphate values etc. and on the presence of some zooplankton as indicator of pollution. A large number of inland fresh water non-culturable fishes and other aquatic biota of the Trimohini Beel may be eliminated in future due to mixing of continuous chemicals from agriculture fields. It is necessary to conserve the ecosystem of Trimohini Beel for the fresh water non-culturable fishes and other aquatic biota.

Key words: Wetland, Agro-chemicals, Zooplankton, Fish disease, Catchment area, Eutrophic nature
\end{abstract}

\section{Introduction}

The wetland ecosystem of Bangladesh is composed of more than 700 rivers, streams, numerous haors, baors, beels, seasonal and perennial floodplain etc. (BBS 1997). During the recent years captive or ponds fishery and beel fishery have become popular in the country. The total number ponds in the country are 12888222 covering an area of 150000 ha. A total of 2832792 ha seasonal floodplain and 1031563 ha is permanent riverine and estuary water body (Nuruzzaman 1990, SPARRSO 1984 and FIB 1986). Through proper culture based fishery and efficient management, an increased fish production of about 16 million m.t./year is possible in inland waters in Bangladesh (Islam 1992).

The agro-chemicals from terrestrial runoff of agricultural fields enter into surface water. This has resulted in the total elimination of a large number of inland fresh water noncultureable fishes and other biota. Ammonia is produced in surface water by decomposition of organic matters and hydrolysis of urea. Acidification and pollution of the surface water are created by $\mathrm{PO}_{4}, \mathrm{SO}_{4}$, chlorides etc. along with insecticides and herbicides, and this acidic polluted water is responsible for fish epizootic ulcerative syndrome (Conway and Pretty 1991, Swarup et al.1992, Bhatt et al. 1999, Bandela et al.1999, Cudchodkar and D'souza 1996 and Islam 2004). Limnological knowledge and their proper applications for better fish yield and management of the ecosystems are prerequisites to sustainable fish sectoral development and maintenance of the eutrophic nature of the wetland ecosystem (Wetzel 1983). Fish health and management of other

\footnotetext{
${ }^{1}$ Corresponding author: Email: aharunc_ku@yahoo.com
} 
biodiversity are practical applications of limnology and aquacultural environment (Khan and Chowdhury 1974 and Toetz 1971). It is most important to study the interrelationship between the physico-chemical aspects and between phyto-zooplankton and the effect of agricultural effluent on the biota. Bhatt et al. (1999), Das et al. (2002), Mishra and Trivedy (1993), Moyle (1946), Rai (1974) studied the limnology and biology of rivers, lakes and wetlands in India. Islam and Khatun (1966), Islam and Nahar (1967), Islam and Shaha (1975) studied the limnology of the polluted waters in Bangladesh. In the recent years environmental scientists of the country are interested to study the limnological status of surface water bodies to assess the water quality and biodiversity for conservation planning of the wetlands. A natural perennial wetland known as Trimohini Beel is situated under Mohonpur upazila of Rajshahi, Bangladesh. Intensive cultivation is being practiced in the catchment area of this wetland and organic, inorganic manures, insecticides, herbicides and fungicides are used at heavy doses, as a result water quality changed (Islam 2004). The available scientific documents in Bangladesh reveal that Tromohini Beel has not been studied up to date. So it is necessary to know the limnological conditions of this wetland for sustainable conservation of aquatic ecosystem. Thus the present investigation has been carried out to find out the physicochemical and zooplankton conditions of Trimohini Beel of Rajshahi for evaluating the pollution level of water of this wetland.

\section{Materials and Methods}

Trimohini wetland is near Rajshahi, Bangladesh. The investigated wetland lies between $24^{\circ} 35^{\prime}$ to $24^{\circ} 40^{\prime} \mathrm{N}$ latitudes and between $88^{\circ} 35^{\prime}$ to $88^{\circ} 40^{\prime} \mathrm{E}$ longitudes (Anon 1997). This perennial wetland is $24 \mathrm{~km}^{2}(8 \mathrm{~km} \times 3 \mathrm{~km})$ and covers an area of $50 \mathrm{~km}^{2}(10 \mathrm{~km} \times 5 \mathrm{~km})$ during monsoon. The catchments area is $150 \mathrm{~km}^{2}(20 \mathrm{~km} \times 7.5 \mathrm{~km})$, where intensive cultivation is being practiced by using organic, inorganic manures, insecticides, herbicides and fungicides. Water and plankton samples were collected from January 2010 to December 2011 in four different spots of the wetlands. Fortnightly samplings were done in this wetland and in each sampling date physico-chemical and biological samples were collected three times ( 8 A.M, 12 Noon and 4 P.M.). An average of these data for each spot was made and average depth of each spot was also determined. Water samples were collected from a depth $10-15 \mathrm{~cm}$ below the surface using a $250 \mathrm{ml}$. BOD bottle. Water colour was detected by following method as stated by Welch (1948). Temperature was noted by a digital thermometer (Model China Empex-range 10-110 ${ }^{\circ} \mathrm{C}$ ). Transparency was determined by a Secchi disc. A digital $\mathrm{pH}$ meter (Model $\mathrm{pH}$ epi HANNA instruments CEEN 50081-1) and portable conductivity meter (Model OSK CM-1K) were used for the measurement of $\mathrm{pH}$ and conductivity respectively. DO content of water was measured by DO meter (Model- JENWAY-9015). Free $\mathrm{CO}_{2}, \mathrm{CO}_{3}, \mathrm{HCO}_{3}$, alkalinity, total hardness, chloride, $\mathrm{BOD}_{5}, \mathrm{COD}$, mobile $\mathrm{NH}_{3}$ and $\mathrm{NH}_{4}-\mathrm{N}$ were determined by following APHA (1989), FAO (1984) and (Welch 1948). Total phosphate, Oxidation-reduction Potential 
(Eh) and Oxidation reduction index $\left(\mathrm{rH}_{2}\right)$ were measured by following Gautam (1990). Primary productivity was measured by Gaarder and Graan (1927).

Plankton were collected by using a plankton net No. 20 silk bolting cloth of mesh size $76 \mu \mathrm{m}$. Identification of plankton was done immediately after collection. Plankton abundance was measured by using a Sedgewick-Rafter counting chamber (Welch 1948) and expressed in unit/1 whether it is an individual or part thereof.

\section{Results and Discussion}

In this study 26 physico-chemical variables were measured and 38 genera of zooplankton were recorded. Average data of 4 spots are presented in Tables 1 and 2 respectively.

During the period of study water colour was found always transparent except monsoon (June to August) in the four study spots. Air temperature was almost similar in all study spots and ranged from $19-33.5^{\circ} \mathrm{C}$ (yearly mean value $29.00 \pm 5.88^{\circ} \mathrm{C}$ ). Water temperature varied from $20-32.5^{\circ} \mathrm{C}$ (yearly mean value $28.29 \pm 4.96^{\circ} \mathrm{C}$ ). Average depth of water was $60-320 \mathrm{~cm}$ (yearly mean value $158.8 \pm 94.6 \mathrm{~cm}$ ). Transparency was $20-110 \mathrm{~cm}$ (yearly mean value $72.08 \pm 29.88 \mathrm{~cm}$ ). TSS varied from $250-380 \mathrm{mg} / 1$ (yearly mean value $273.3 \pm 49.92 \mathrm{mg} / \mathrm{l})$ in all spots. Electric conductivity ranged from $84.6-110.5 \mu \mathrm{mho} / \mathrm{cm}$ (yearly mean value $93.65 \pm 10.15 \mu \mathrm{mho} / \mathrm{cm}$ ) in all spots. $\mathrm{pH}$ varied from $7.2-7.5$ (yearly mean value $7.35 \pm 0.10$ ). Free $\mathrm{CO}_{2}$ ranged from $13-23 \mathrm{mg} / \mathrm{l}$ (yearly mean value $17.50 \pm 3.23$ $\mathrm{mg} / \mathrm{l}) . \mathrm{CO}_{3}$ alkalinity was nil in all spots and $\mathrm{HCO}_{3}$ alkalinity varied from $45-72 \mathrm{mg} / \mathrm{l}$ (yearly mean value $61.20 \pm 11.75 \mathrm{mg} / \mathrm{l}$ ). Ca-hardness and $\mathrm{Mg}$-hardness ranged from 75.0$85.0 \mathrm{mg} / \mathrm{l}$ (yearly mean value $80.28 \pm 3.53 \mathrm{mg} / \mathrm{l}$ ) and $40.0-52.4 \mathrm{mg} / \mathrm{l}$ (yearly mean value $48.12 \pm 3.96 \mathrm{mg} / \mathrm{l})$ respectively in all spots. Total hardness varied from $120-137 \mathrm{mg} / \mathrm{l}$ (yearly mean value $128.40 \pm 7.03 \mathrm{mg} / \mathrm{l}$ ). Chloride ranged from $60-100 \mathrm{mg} / 1$ (yearly mean value $78.79 \pm 12.62 \mathrm{mg} / \mathrm{l}$ ). DO values varied from $5.3-5.7 \mathrm{mg} / 1$ (yearly mean value $5.57 \pm 0.16 \mathrm{mg} / \mathrm{l}$ ) and percentage of sat. of oxygen ranged from 64.5-78.2 (yearly mean value 71.66 \pm 5.14 ) in all spots. BOD and COD values varied from 5.9-6.4 mg/l (yearly mean value $6.21 \pm 0.16 \mathrm{mg} / \mathrm{l}$ ) and $13.70-14.64 \mathrm{mg} / \mathrm{l}$ (yearly mean value $14.28 \pm 0.30 \mathrm{mg} / \mathrm{l}$ ) respectively. Nitrite-nitrogen and $\mathrm{NH}_{4}-\mathrm{N}$ ranged from $0.15-0.59 \mathrm{mg} / 1$ (yearly mean value $0.39 \pm 0.16 \mathrm{mg} / \mathrm{l}$ ) and $0.15-0.36 \mathrm{mg} / 1$ (yearly mean value $0.28 \pm 0.08 \mathrm{mg} / \mathrm{l}$ ) respectively. $\mathrm{PO}_{4}$ values varied from $0.20-0.45 \mathrm{mg} / \mathrm{l}$ (yearly mean value $0.33 \pm 0.07 \mathrm{mg} / \mathrm{l}$ ). Eh and $\mathrm{rH}_{2}$ values were from $0.28-0.32 \mathrm{mv}$ (yearly mean value $0.30 \pm 0.01 \mathrm{mv}$ ) and 24.48-25.40 (yearly mean value $25.02 \pm 0.31$ ) respectively. Gross primary productivity (GPP) and net primary productivity (NPP) ranged from $0.016-0.020 \mathrm{mgC} / \mathrm{h} / 1$ (yearly mean value $0.018 \pm 0.001 \mathrm{mgC} / \mathrm{h} / \mathrm{l}$ ) and $0.008-0.011 \mathrm{mgC} / \mathrm{h} / 1$ (yearly mean value $0.009 \pm 0.0012$ $\mathrm{mgC} / \mathrm{h} / \mathrm{l})$ respectively in all spots. 
Table1. Average Physico-chemical conditions of all study spots of Trimohini wetland, Mahonpur of Rajshahi.

\begin{tabular}{|c|c|c|c|c|c|c|c|c|c|c|c|c|c|}
\hline Parameters & Jan. & Feb. & Mar. & April. & May & June & July & Aug. & Sep. & Oct. & Nov. & Dec. & Mean \pm SD \\
\hline $\begin{array}{l}\text { Air Temp. } \\
{ }^{\circ} \mathrm{C}\end{array}$ & 19 & 20 & 32.5 & 33 & 33 & 32.5 & 32.5 & 33 & 33.5 & 32 & 27 & 20 & $29.00 \pm 5.88$ \\
\hline $\begin{array}{l}\text { Water } \\
\text { Temp. }{ }^{\circ} \mathrm{C}\end{array}$ & 20 & 21 & 30 & 32 & 32 & 31 & 31 & 32 & 32.5 & 30 & 28 & 20 & $28.29 \pm 4.96$ \\
\hline $\begin{array}{l}\text { Av. Depth } \\
\text { (cm) }\end{array}$ & 110 & 100 & 85 & 75 & 60 & 85 & 300 & 320 & 270 & 230 & 150 & 120 & $158.8 \pm 94.6$ \\
\hline $\begin{array}{l}\text { S.D. Depth } \\
(\mathrm{cm})\end{array}$ & 110 & 100 & 85 & 75 & 60 & 50 & 20 & 20 & 70 & 80 & 95 & 100 & $72.08 \pm 29.88$ \\
\hline T.S.S (mg/l) & 250 & 250 & 250 & 255 & 250 & 260 & 380 & 380 & 255 & 250 & 250 & 250 & $273.3 \pm 49.92$ \\
\hline $\begin{array}{l}\text { E. Con } \\
(\mu \mathrm{moh} / \mathrm{cm})\end{array}$ & 110.5 & 108.5 & 90.6 & 86.6 & 86.6 & 88.6 & 88.6 & 86.6 & 84.6 & 86.6 & 95.5 & 110.5 & $93.65 \pm 10.15$ \\
\hline pH & 7.2 & 7.3 & 7.3 & 7.4 & 7.5 & 7.4 & 7.4 & 7.4 & 7.5 & 7.3 & 7.3 & 7.2 & $7.35 \pm 0.10$ \\
\hline $\begin{array}{l}\text { Free } \mathrm{CO}_{2} \\
(\mathrm{mg} / \mathrm{l})\end{array}$ & 20 & 20 & 15 & 15 & 13 & 13 & 20 & 23 & 15 & 18 & 18 & 20 & $17.50 \pm 3.23$ \\
\hline $\mathrm{CO}_{3}(\mathrm{mg} / \mathrm{l})$ & 0 & 0 & 0 & 0 & 0 & 0 & 0 & 0 & 0 & 0 & 0 & 0 & 0 \\
\hline $\mathrm{HCO}_{3}(\mathrm{mg} / \mathrm{l})$ & 72 & 72 & 70 & 50 & 45 & 48 & 48 & 49 & 68.4 & 70 & 70 & 72 & $61.20 \pm 11.75$ \\
\hline $\begin{array}{l}\text { Ca hard } \\
(\mathrm{mg} / \mathrm{l})\end{array}$ & 76 & 78 & 81 & 82 & 83 & 83 & 80 & 81 & 84.4 & 85 & 75 & 75 & $80.28 \pm 3.53$ \\
\hline $\begin{array}{l}\text { Mg hard } \\
(\mathrm{mg} / \mathrm{l})\end{array}$ & 44 & 47 & 49 & 50 & 52 & 52 & 40 & 49 & 52.4 & 52 & 45 & 45 & $48.12 \pm 3.96$ \\
\hline $\begin{array}{l}\text { Total hard } \\
(\mathrm{mg} / \mathrm{l})\end{array}$ & 120 & 125 & 130 & 132 & 135 & 135 & 120 & 130 & 136.8 & 137 & 120 & 120 & $128.40 \pm 7.03$ \\
\hline & 75 & 77 & 65 & 63 & 60 & 88 & 95 & 100 & 87.5 & 85 & 75 & 75 & $78.79 \pm 12.62$ \\
\hline DO (mg/l) & 5.7 & 5.6 & 5.5 & 5.4 & 5.4 & 5.8 & 5.7 & 5.7 & 5.3 & 5.4 & 5.6 & 5.7 & $5.57 \pm 0.16$ \\
\hline $\begin{array}{l}\% \text { of Sat. of } \\
\mathrm{O}_{2}\end{array}$ & 64.5 & 64.5 & 73.04 & 73.8 & 73.8 & 78.2 & 76.8 & 77.90 & 72.90 & $\begin{array}{c}67.7 \\
0\end{array}$ & $\begin{array}{c}72.3 \\
0\end{array}$ & 64.50 & $71.66 \pm 5.14$ \\
\hline $\mathrm{BOD}_{5}(\mathrm{mg} / \mathrm{l})$ & 6.2 & 6.3 & 6.2 & 6.1 & 6.0 & 5.9 & 6.3 & 6.3 & 6.4 & 6.1 & 6.4 & 6.3 & $6.21 \pm 0.16$ \\
\hline COD (mg/l) & 14.26 & 14.45 & 14.26 & 14.07 & 13.88 & 13.70 & 14.45 & 14.45 & 14.64 & $\begin{array}{c}14.0 \\
7\end{array}$ & $\begin{array}{c}14.6 \\
4\end{array}$ & 14.45 & $14.28 \pm 0.30$ \\
\hline $\begin{array}{l}\text { Nitrite- } \\
\text { nitrogen } \\
(\mathrm{mg} / \mathrm{l})\end{array}$ & 0.30 & 0.30 & 0.25 & 0.20 & 0.15 & 0.58 & 0.59 & 0.58 & 0.56 & 0.50 & 0.40 & 0.30 & $0.39 \pm 0.16$ \\
\hline $\begin{array}{l}\mathrm{NH}_{4}-\mathrm{N} \\
(\mathrm{mg} / \mathrm{l})\end{array}$ & 0.35 & 0.30 & 0.15 & 0.15 & 0.20 & 0.25 & 0.25 & 0.30 & 0.35 & 0.36 & 0.36 & 0.36 & $0.28 \pm 0.08$ \\
\hline $\mathrm{PO}_{4}(\mathrm{mg} / \mathrm{l})$ & 0.30 & 0.30 & 0.25 & 0.25 & 0.20 & 0.35 & 0.40 & 0.45 & 0.40 & 0.35 & 0.35 & 0.30 & $0.33 \pm 0.07$ \\
\hline Eh (mv) & 0.32 & 0.32 & 0.30 & 0.29 & 0.28 & 0.30 & 0.30 & 0.30 & 0.28 & 0.30 & 0.31 & 0.32 & $0.30 \pm 0.01$ \\
\hline $\mathrm{rH}_{2}$ & 25.4 & 25.35 & 25.09 & 24.48 & 24.63 & 25.04 & 25.05 & 25.05 & 24.58 & $\frac{25.0}{5}$ & $\begin{array}{c}25.1 \\
5\end{array}$ & 25.40 & $25.02 \pm 0.31$ \\
\hline $\begin{array}{l}\text { GPP } \\
(\mathrm{mgC} / \mathrm{h} / \mathrm{l})\end{array}$ & 0.018 & 0.018 & 0.019 & 0.020 & 0.020 & 0.016 & 0.017 & 0.017 & 0.020 & $\begin{array}{c}0.02 \\
0\end{array}$ & $\begin{array}{c}0.01 \\
8\end{array}$ & 0.018 & $0.018 \pm 0.001$ \\
\hline $\begin{array}{l}\mathrm{NPP} \\
(\mathrm{mgC} / \mathrm{h} / \mathrm{l})\end{array}$ & 0.009 & 0.009 & 0.010 & 0.011 & 0.011 & 0.008 & 0.008 & 0.008 & 0.010 & $\begin{array}{c}0.01 \\
1\end{array}$ & $\begin{array}{c}0.00 \\
9\end{array}$ & 0.009 & $0.009 \pm 0.001$ \\
\hline
\end{tabular}

In total 38 zooplankton genera were recorded from four spots during the period of study of which 12 genera belonged to Copepoda (31.58\%), 12 to Cladocera (31.58\%) and 14 to 
Rotifer (36.84\%) (Table 2). The average zooplankton abundance of four spots varied from 15015-26000 units/1 with mean abundance (21240 units/1).

The abundance of Copepoda, Cladocera and Rotifera varied from 6740-11170 units/1 (mean abundance 9138 units/1; 43.02\%), 3730-6565 units/1 (mean abundance 5418 units/1; 25.51\%) and 4545-8265 units/1 (mean abundance 6684 units/1; 31.47\%) respectively.

The Copepoda were Allodiaptomus sp., Cyclops sp., Diaptomus sp., Eucyclops sp., Heliodiaptomus sp., Paradiatomus sp., Phyllodiaptomus sp., Rhinediaptomus sp., Macrocyclops sp., Mesocyclops sp.,Orthocyclops sp. and Paracyclops sp.. Diaptomus sp. (22.28\%) and Cyclops sp. $(20.46 \%)$ were found to occur in higher abundance followed by Eucyclops sp. (12.22\%), Mesocyclops sp. (9.83\%) and others.

The Cladocerans were represented by Alona sp., Alonella sp., Bosmina sp., Bosminopsis sp. Ceriodaphnia sp., Daphnia sp., Diaphanosoma sp., Macrothrix sp., Moina sp., Polyphemus sp., Sida sp. and Simocephalus sp.. The Cladoceran were dominated by Diaphanosoma sp.(10.50\%), Simocephalus sp. (10.27\%), Daphnia sp. (9.89\%) and others.

The Rotifers were represented by Brachionus sp., Dorystoma sp., Filinia sp., Gastropus sp., Harringia sp., Hexarthra sp., Keratella sp., Monostyla sp., Notholca sp., Philodina sp., Platyias sp., Polyarthra sp., Scaridium sp. and Trichocerca sp.. The Rotiferans were dominated by Trichocerca sp.(11.20\%), Keratella sp. (10.86\%), Brachionus sp. $(9.91 \%)$ and others.

The Rotifers are tolerant to varying degree of physico-chemical and biological conditions. Islam et al. (2005), Islam et al. (2001) and Arora (1966) observed that the rotifers occur in eutrophic waters in high abundance. Islam et al. (2005), Islam et al. (2001), Arora (1966) and Bergins (1949) designated a large number of rotifers genera including Barchionus sp., Keratella sp., Filinia sp. Gastropus sp., Hexarthra sp., Polyarthra sp., Trichocerca sp. and others as indicators of eutrophic or polluted waters and the findings of the present study appears to be in conformity with their works.

Air and water temperature was always found with low values. Indiscriminately uses of agro-chemicals like urea, TSP, chloride in catchment area of the wetland caused the water of the wetland acidic (Islam 2004). In unpolluted rivers the values of chloride are usually low (between 2 to $10 \mathrm{mg} / \mathrm{l}$ ). The desirable level of chloride in water should be below $200 \mathrm{mg} / \mathrm{l}$ for human consumption (Koshy and Nayar 1999). In the studied wetland the chloride content showed higher values. The higher amounts of nitrite nitrogen $\left(\mathrm{NO}_{2}-\right.$ $\mathrm{N}$ ) and ammonium nitrogen $\left(\mathrm{NH}_{4}-\mathrm{N}\right)$ indicated the higher loads of organic matters and excessive use of inorganic fertilizers which was washed into natural water by the rain and flood water (Islam 2004 and Swarup et al. 1992). Low pH, Eh, DO and high $\mathrm{rH}_{2}, \mathrm{BOD}$, $\mathrm{COD}$ are the indication of presence of both organic and inorganic load in study water coupled with $\mathrm{NH}_{4}-\mathrm{N}, \mathrm{NO}_{2}-\mathrm{N}$ and $\mathrm{PO}_{4}$ values and the whole physical-chemical variable 
together formed a complex of nutrient status of biological importance imparting an eutrophic nature to study water (Morris and Stumm 1967, Morrissette and Mavinic 1978, Lakshminarayana 1965 and Jayangaudar 1964).

Table 2. Monthly average of abundance (units/l) of zooplankton of Trimohini wetland.

\begin{tabular}{|c|c|c|c|c|c|c|c|c|c|c|c|c|c|c|}
\hline Zooplankton & Jan & Feb & Mar & Apr & May & Jun & Jul & Aug & Sep & Oct & Nov & Dec & $\begin{array}{l}\text { Yearly } \\
\text { Mean }\end{array}$ & $\%$ \\
\hline \multicolumn{15}{|l|}{ Copepoda } \\
\hline $\begin{array}{l}\text { Allodiaptom } \\
\text { us 2sps. }\end{array}$ & 275 & 270 & 265 & 275 & 280 & 290 & 285 & 250 & 195 & 175 & 200 & 230 & 249 & 2.73 \\
\hline $\begin{array}{l}\text { Cyclops } \\
\text { 3sps. }\end{array}$ & 1800 & 2000 & 1890 & 2050 & 2100 & 2150 & 2100 & 2000 & 1650 & 1500 & 1590 & 1600 & 1869 & 20.46 \\
\hline $\begin{array}{l}\text { Diaptomus } \\
2 \text { sps. }\end{array}$ & 1900 & 2050 & 2200 & 2250 & 2300 & 2400 & 2350 & 1800 & 1775 & 1650 & 1750 & 2000 & 2035 & 22.28 \\
\hline $\begin{array}{l}\text { Eucyclops } \\
2 \text { sps. }\end{array}$ & 1000 & 1120 & 1200 & 1250 & 1300 & 1400 & 1300 & 1200 & 1100 & 800 & 825 & 900 & 1116 & 12.2 \\
\hline $\begin{array}{l}\text { Heliodiapto } \\
\text { mus sp. }\end{array}$ & 450 & 475 & 500 & 550 & 575 & 600 & 575 & 500 & 450 & 350 & 375 & 400 & 483 & 5.29 \\
\hline $\begin{array}{l}\text { Paradiapto } \\
\text { mus sp. }\end{array}$ & 295 & 300 & 320 & 350 & 375 & 400 & 350 & 300 & 250 & 195 & 200 & 260 & 300 & 3.28 \\
\hline $\begin{array}{l}\text { Phyllodiapt } \\
\text { omus sp. }\end{array}$ & 300 & 250 & 350 & 370 & 380 & 400 & 385 & 300 & 270 & 200 & 230 & 270 & 309 & 3.38 \\
\hline $\begin{array}{l}\text { Rhinediapto } \\
\text { mus sp. }\end{array}$ & 290 & 320 & 340 & 350 & 380 & 430 & 350 & 325 & 275 & 230 & 240 & 250 & 315 & 3.45 \\
\hline $\begin{array}{l}\text { Macrocyclo } \\
\text { ps sp. }\end{array}$ & 900 & 940 & 960 & 980 & 1000 & 1050 & 950 & 900 & 600 & 500 & 540 & 870 & 849 & 9.29 \\
\hline $\begin{array}{l}\text { Mesocyclps } \\
\text { sp. }\end{array}$ & 100 & 1020 & 1050 & 1090 & 1120 & 1150 & 1000 & 950 & 900 & 700 & 750 & 950 & 898 & 9.83 \\
\hline $\begin{array}{l}\text { Orthocyclop } \\
s \text { sp. }\end{array}$ & 290 & 300 & 310 & 350 & 350 & 320 & 300 & 250 & 195 & 160 & 200 & 250 & 273 & 2.99 \\
\hline $\begin{array}{l}\text { Paracyclops } \\
\text { sp. }\end{array}$ & 400 & 470 & 500 & 550 & 570 & 580 & 490 & 400 & 350 & 280 & 295 & 400 & 440 & 4.82 \\
\hline Total & 8000 & 9515 & 9885 & $\begin{array}{c}1041 \\
5\end{array}$ & 10730 & 11170 & 10435 & 9175 & 8010 & 6740 & 7195 & 8380 & 9138 & 100 \\
\hline \multicolumn{15}{|l|}{ Cladocera } \\
\hline Alona 2sps. & 360 & 355 & 370 & 380 & 390 & 340 & 300 & 290 & 270 & 210 & 290 & 350 & 325 & 6.01 \\
\hline $\begin{array}{l}\text { Alonella } \\
\text { 2sps. }\end{array}$ & 390 & 385 & 395 & 430 & 450 & 470 & 450 & 430 & 340 & 290 & 330 & 400 & 397 & 7.32 \\
\hline $\begin{array}{l}\text { Bosmina } \\
2 \text { sps. }\end{array}$ & 410 & 420 & 450 & 470 & 490 & 500 & 495 & 445 & 395 & 300 & 390 & 440 & 434 & 8.01 \\
\hline $\begin{array}{l}\text { Bosminopsis } \\
2 \text { sps. }\end{array}$ & 390 & 380 & 400 & 430 & 450 & 470 & 490 & 400 & 350 & 320 & 350 & 345 & 398 & 7.34 \\
\hline $\begin{array}{l}\text { Ceriodaphni } \\
\text { a sp. }\end{array}$ & 400 & 430 & 450 & 470 & 490 & 520 & 490 & 410 & 370 & 330 & 370 & 400 & 428 & 7.89 \\
\hline $\begin{array}{l}\text { Daphnia } \\
\text { 2sps. }\end{array}$ & 500 & 540 & 580 & 600 & 620 & 670 & 620 & 600 & 400 & 340 & 460 & 490 & 535 & 9.87 \\
\hline $\begin{array}{l}\text { Diaphanoso } \\
\text { ma } 2 \text { sps. }\end{array}$ & 550 & 590 & 620 & 640 & 670 & 690 & 640 & 610 & 420 & 370 & 495 & 530 & 569 & $\begin{array}{c}10.5 \\
0\end{array}$ \\
\hline $\begin{array}{l}\text { Macrothrix } \\
2 \text { sps. }\end{array}$ & 540 & 570 & 600 & 620 & 640 & 680 & 610 & 500 & 400 & 350 & 410 & 450 & 531 & 9.80 \\
\hline Moina 3sps. & 380 & 390 & 420 & 450 & 480 & 500 & 440 & 400 & 360 & 300 & 340 & 345 & 400 & 7.39 \\
\hline
\end{tabular}


(Contd.)

\begin{tabular}{|c|c|c|c|c|c|c|c|c|c|c|c|c|c|c|}
\hline Zooplankton & Jan & Feb & Mar & Apr & May & Jun & Jul & Aug & Sep & Oct & Nov & Dec & $\begin{array}{l}\text { Yearly } \\
\text { Mean }\end{array}$ & $\%$ \\
\hline $\begin{array}{l}\text { Polyphemus } \\
\text { 2sps. }\end{array}$ & 500 & 490 & 520 & 540 & 580 & 600 & 550 & 520 & 440 & 320 & 480 & 500 & 503 & 9.29 \\
\hline Sida 2sps. & 300 & 330 & 350 & 380 & 400 & 430 & 400 & 350 & 300 & 250 & 295 & 320 & 342 & 6.31 \\
\hline $\begin{array}{l}\text { Simocephal } \\
\text { us } 2 \text { sps. }\end{array}$ & 520 & 540 & 580 & 600 & 640 & 695 & 640 & 600 & 500 & 350 & 495 & 515 & 556 & $\begin{array}{c}10.2 \\
7\end{array}$ \\
\hline Total & 5240 & 5420 & 5735 & 6010 & 6300 & 6565 & 6125 & 5555 & 4545 & 3730 & 4705 & 5085 & 5418 & 100 \\
\hline \multicolumn{15}{|l|}{ Rotifera } \\
\hline $\begin{array}{l}\text { Brachionus } \\
\text { 3sps. }\end{array}$ & 640 & 650 & 690 & 720 & 750 & 800 & 760 & 700 & 600 & 420 & 590 & 630 & 663 & 9.91 \\
\hline $\begin{array}{l}\text { Dorystoma } \\
2 \text { sps. }\end{array}$ & 400 & 420 & 450 & 490 & 520 & 580 & 500 & 470 & 400 & 370 & 400 & 430 & 453 & 6.77 \\
\hline Filinia 2sps. & 390 & 400 & 430 & 460 & 500 & 580 & 500 & 450 & 400 & 300 & 370 & 400 & 432 & 6.46 \\
\hline $\begin{array}{l}\text { Gastropus } \\
\text { 2sps. }\end{array}$ & 460 & 480 & 500 & 530 & 560 & 600 & 550 & 500 & 450 & 390 & 450 & 475 & 495 & 7.41 \\
\hline $\begin{array}{l}\text { Harringia } \\
2 \text { sps. }\end{array}$ & 450 & 425 & 440 & 460 & 495 & 620 & 490 & 400 & 370 & 300 & 430 & 440 & 443 & 6.63 \\
\hline $\begin{array}{l}\text { Hexarthra } \\
2 \text { sps. }\end{array}$ & 330 & 360 & 390 & 400 & 430 & 470 & 400 & 320 & 295 & 240 & 320 & 330 & 357 & 5.34 \\
\hline $\begin{array}{l}\text { Keratella } \\
3 \text { sps. }\end{array}$ & 700 & 730 & 760 & 790 & 820 & 850 & 790 & 720 & 650 & 440 & 740 & 720 & 726 & $\begin{array}{c}10.8 \\
6\end{array}$ \\
\hline $\begin{array}{l}\text { Monostyla } \\
\text { sp. }\end{array}$ & 250 & 270 & 290 & 310 & 330 & 370 & 370 & 300 & 250 & 230 & 225 & 260 & 288 & 4.31 \\
\hline $\begin{array}{l}\text { Notholca } \\
\text { 2sps. }\end{array}$ & 220 & 240 & 270 & 300 & 360 & 400 & 330 & 300 & 200 & 195 & 210 & 220 & 270 & 4.05 \\
\hline $\begin{array}{l}\text { Philodina } \\
2 \text { sps. }\end{array}$ & 320 & 350 & 370 & 390 & 420 & 450 & 400 & 370 & 295 & 250 & 300 & 340 & 355 & 5.30 \\
\hline $\begin{array}{l}\text { Platyias } \\
\text { 3sps. }\end{array}$ & 450 & 590 & 600 & 630 & 670 & 695 & 650 & 550 & 450 & 350 & 420 & 450 & 542 & 8.11 \\
\hline $\begin{array}{l}\text { Polyarthra } \\
\text { 2sps. }\end{array}$ & 345 & 320 & 360 & 390 & 450 & 480 & 400 & 390 & 290 & 240 & 500 & 530 & 391 & 5.85 \\
\hline $\begin{array}{l}\text { Scaridium } \\
2 \mathrm{sps} .\end{array}$ & 500 & 530 & 580 & 600 & 640 & 680 & 520 & 420 & 390 & 320 & 520 & 550 & 521 & 7.79 \\
\hline $\begin{array}{l}\text { Trichocerca } \\
\text { 3sps. }\end{array}$ & 750 & 725 & 750 & 800 & 840 & 690 & 920 & 840 & 750 & 500 & 700 & 720 & 749 & $\begin{array}{c}11.2 \\
0\end{array}$ \\
\hline Total & 6205 & 6490 & 6880 & 7270 & 7785 & 8265 & 7580 & 6730 & 5790 & 4545 & 6175 & 6495 & 6684 & 100 \\
\hline Grand Total & 19445 & 21425 & 22500 & $\begin{array}{c}2369 \\
5\end{array}$ & 24815 & 26000 & 24140 & 21460 & 18345 & $\begin{array}{c}1501 \\
5\end{array}$ & 18075 & $\begin{array}{c}1996 \\
0\end{array}$ & $\begin{array}{c}2124 \\
0\end{array}$ & \\
\hline
\end{tabular}

The GPP and NPP values, higher amounts of $\mathrm{CO}_{2}$ and absence of $\mathrm{CO}_{3}$ throughout the study period indicate the low rate of photosynthesis and high rate of respiration by the aquatic biota in Trimihini Beel. Similar findings were recorded by Islam (2004), Bhatt et al. (1999) and Das et al. (2002) in their studies. Islam (2004) also observed that low air and water temperature, low $\mathrm{pH}$ etc. are the causes of epizootic ulcerative syndrome of fin fishes in natural wetlands. Higher amounts of nitrite nitrogen $\left(\mathrm{NO}_{2}-\mathrm{N}\right)$ and ammonium nitrogen $\left(\mathrm{NH}_{4}-\mathrm{N}\right)$ were recorded in this wetland, which are the causes of death of more than hundred species of fish (Cudchodkar and D'souza 1996, Islam et al. 2001 and Islam 2004). Islam (2004) mentioned that toxic chemicals including fungicides, insecticides, 
herbicides, urea, chlorides, sulphates and phosphates were used at heavy doses in agricultural crops fields around the studied wetland; as a result residual effluent of used chemicals or as direct solution of chemicals entered into the water of wetland.

According to Gautam (1990), Chowdhury et al. (1996), Islam and Nahar (1967), Lakshiminarayana (1965), Jayangauder (1964), Zafar (1964), George (1968), Moyle (1946), Montgomery et al. (1964), Mortimer (1956), Islam et al. (1998), Zaman et al. (1993), Mishra et al. (1992) and Ameen et al. (1986) the studied wetland marked as a medium level polluted wetland on the basis of values of the redox characteristics i.e. $\mathrm{pH}$, DO, BOD, COD, Eh, and $\mathrm{rH}_{2}$ chlorides, nitrites, ammonium, phosphate values etc. and on the presence of some zooplankton as pollution indicator. The findings of the present study indicate that a large number of inland fresh water non-culturable fishes and other aquatic biota of the Trimohini Beel may be eliminated in future due to mixing of continuous chemicals from agriculture fields. Toxic chemicals have a deleterious effect on the wetlands ecosystem as a whole (Cudchodkar and D'souza 1996, Chowdhury et al. 1996 and Gautam 1990). So, it is necessary to conserve the ecosystem of Trimohini Beel for the fresh water non-culturable fishes and other aquatic biota. A sustainable management plan as well as area demarcation is necessary for the agriculture to protect the further degradation of water quality and biodiversity of the study area. Findings of this study will be helpful for further study and sustainable management plan of the fresh water wetlands.

\section{Acknowledgment}

The authors are grateful to Professor Dr. Md. Altaf Hossain, Dept. of Zoology, Rajshahi University and Prof. M. Zaman, Dept. of Botany, Rajshahi University for their cooperations.

\section{Reference}

Ameen, M., Z. N. T. Begum, S. Ali, M. M. Rahman and T.K. Roy. 1986. A comparative Limnological study of two fish ponds in Raipur, Dhaka Uni. stud. part E 1(1): 25-34.

Anon. 1997. Bhumi O Mrittika Sampod Babohar Nirdeshika, Mohanpur Thana, Rajshahi District, SRDI, coordinated by BARC. S-210: 1-122.

APHA. 1989. Standard methods for the examination of water and waste water. American Public Health Association, Washington. 1125 pp.

Arora, H.C. 1966. Rotifer as indicators of trophic nature of environment. Hydrobiologia 32(1-2): 146-159.

Bandela, N.N., D. P. Vaidya, V. S. Lomte and S.V. Shivanikar. 1999. The distribution pattern of phosphate and nitrogen forms and their interrelationships in Barul dam water, India. Poll. Res.18 (4): 411-414.

BBS. 1997. Statistical yearbook of Bangladesh, BBS, 18th edi. p 1-690.

Bergins, B. 1949. Zur Lomnologe der seen sudostlent lend. Schweigh. Hydrobiologia 2(11): 583607.

Bhatt, L.R., P. Lacoul, H. D. Lekhak, and P.K. Jha. 1999. Physico-chemical characteristics and phytoplankton of Toudaha Lake, Katmandu. Poll. Res. 18(4): 353-358. 
Chowdhury. A.H., S. Naz. and M. Zaman. 1996. Effect of industrial effluents on the physicochemical and biological conditions of Padma water near Rajshahi. J. Bio. Sci. 4: 9-19.

Conway, G.R. and J.N. Pretty. 1991. Pollution and farming system, JAFSA 1 (1): 29-53.

Cudchodkar, S. and J. D'souza. 1996. Studies on the impact of chemical pollutants on fish and fish diseases in Goan estuaries. J. Inddus. Poll.Cont. 12(2): 109-117.

Das, D., P. Dev, M. Talukder, S. D. Gupta, S. Gupta and A. Gupta. 2002. Ecological issues in the floodplain wetlands of Barak valley, Assam, India: A holistic appraisal. Wetlands, People, Land, Water and Fish: 31-32.

FAO. 1984. Physical and chemical methods of soil and water analysis. FAO 10: 1-275.

FIB. 1986. Fisheries information Bulletin. Water area statistics of Bangladesh, Directorate of Fisheries. FAO \& UNDP 3(1): 1-33.

Gaarder, T. and H.H. Graan. 1927. Investigation of the production of plankton in Oslo Fjord Rapp. Proc. Verb. Cons. Perm. Inter Explor. Mer.: 421-448.

Gautam, A.1990. Ecology and pollution of mountain water. Ashish publication, India. 209pp.

George, M.M. 1968. Seasonal trend in physico-chemical factors and plankton of fresh water fish ponds. Hydrobiologia 13: 203-230.

Islam, A.K.M.N. and M. Khatun. 1966. Preliminary studies of the phytoplankton of polluted waters. Sci. Res. 3(2): 94-109.

Islam, A.K.M.N. and L. Nahar. 1967. Preliminary studies on the phytoplankton of polluted waters, ii Blue green algae. Sci. Res. 4(2-3): 141-149.

Islam, A.K.M.N. and J. Shaha. 1975.Limnological Studies of the Ramna Lake at Dhaka. Dhaka Uni. Stud. 23B(2): 31-37.

Islam M.A. 1992. Kumargara Jalashayer Mathsa Sampad O Tar Babasthapona, M.Sc. Thesis, Dept. Zool., Raj. Uni. 68pp.

Islam, M.A. 2004. Investigation on the plankton biota of Barind wetland at Mohanpur in relation to its physico-chemical conditions. Ph.D. Thesis, Dept. Bot., Raj Uni.163pp.

Islam, M.A., A. H. Chowdhury and M. Zaman. 1998. Seasonal occurrence of zooplankton in four managed fish ponds in Rajshahi, Uni. J. Zool., Raj. Uni. 17:51-60.

Islam, M.A., A. H. Chowdhury and M. Zaman. 2001. Limnology of fish ponds in Rajshahi, Bangladesh. Ecology \& Environment 7(1): 1-7.

Islam, M.A., A. H. Chowdhury, M. A. Hossain and M. Zaman. 2005. Biological control harmful zooplankton in nursery fish ponds. Ecology \& Environ. 13(3-4): 505-509.

Jayangaudar, I. 1964. The Bio-Ecological study of Nuggikari lake in Dharwas, Mysore state, South India. Hydrobiologia 23(3-4): 515-532.

Khan, M.A.G. and S.H. Chowdhury. 1974. Physical and chemical limnology of lake Kaptai, Bangladesh. Trop. \& Ecol. 35(1): 35-51.

Koshy, M. and T.V. Nayar. 1999. Water quality aspects Pamba. Poll. Res. 18(4): 501-510.

Lakshminarayana, J. 1965. Studies on the phytoplankton of the river Ganges, Varanasi, India, ii. The seasonal growth and succession of planktonic algae in the river Ganges. Hydrobiologia 25:138-165.

Mishra, P.C. and R.K. Trivedy. 1993. Ecology of pollution of Indian lakes and reservoirs. Ashish Pub. House, New Delhi, India.

Mishra, S.N., R. Swarup and V.P. Jauhari. 1992. Encyclopedia of Ecology, Environment and pollution control. Enviro. Air. \& Water Analy. Pub. 317-337 pp.

Montogomary, H.A.C., N. S. Thom and A. Cockburn. 1964. Determination of dissolved Oxygen by the Winkler method and solubility of Oxygen in pure water and Sea water. J. Appl. Chem. 14: 280-296.

Morris, J.C. and W. Stumm. 1967. Redox equilibrium and measurements of potentials in the aquatic environment. Adv. Chem. Seri. 67: 270-285.

Morrissette, D.G. and D.S. Mavinic. 1978. BOD test variable. J. Engin. Dev. E.P. 6: 1213-1222. 
Mortimer, C.H. 1956. The Oxygen content of air saturated fresh waters and aids in calculation percentage saturation, Mitt. Int. Verein. Theor. Ange. Limno. No. 6.

Moyle, J.B. 1946. Some indices of lake productivity, Trans. Amer. Fish. SOC. 76: 322-334.

Nuruzzaman, A.K.M. 1990. Perspectives on fisheries development in Bangladesh, BARC. P:1198.

Rai, H. 1974. Limnological studies on river Yamuna at Delhi, India. Arch. Hydrob. 73(3): 369-393.

SPARRSO. 1984. Bangladesh space research and remote sensing Organization, FAO, UNDP, FRSS, Directorate of Fisheries.

Swarup, R., S. N. Mishra and V.P. Jauhari. 1992. Encyclopedia of Ecology, Environment and pollution control. Environment, Air and Water Analysis 17: 1-136.

Toetz, D.W. 1971. Effect of pH, phosphate and ammonia on the rate of uptake of nitrate and ammonia by fresh water phytoplankton. Ecology, 52(4): 903-908.

Welch, P.S. 1948. Limnological Methods, McGraw Hill Book Company, New York. 381pp.

Wetzel, R.G. 1983. Limnology, 2nd ed., Sounders College Publishing, USA. 767 pp.

Zafar, A.R. 1964. On the Ecology of algae in certain fish ponds in Hydarabad, India. Physico-chemical complexes. Hydrobiologia 23: 179-195.

Zaman, L., M. Khandker and M.R. Nabi. 1993. A comparative limnology of three ponds in Jahangirnagar University campus, physical and chemical aspects. Bang. J. Bot. 22(1): 8187. 